\title{
Effects of Xylanase in Corn- or Wheat-Based Diets on Cecal Microbiota of Broilers
}

\author{
Jian Wang ${ }^{1}$, Heng Cao ${ }^{1}$, Chengling Bao', Yajing Liu' ${ }^{1}$, Bing Dong ${ }^{1}$, Chunlin Wang ${ }^{1}$, \\ Zhenda Shang ${ }^{2}$, Yunhe Cao ${ }^{1,2 *}$ and Suozhu Liu ${ }^{2 *}$
}

'State Key Laboratory of Animal Nutrition, College of Animal Science and Technology, China Agricultural University, Beijing, China, ${ }^{2}$ College of Animal Science, Tibet Agricultural and Animal Husbandry University, Linzhi, China

Xylanase has been demonstrated to improve growth performance of broilers fed wheator corn-based diets due to its ability to degrade arabinoxylans (AX). However, content and structure of $A X$ in corn and wheat are different, comparing effects of xylanase on cecal microbiota of broilers fed corn- or wheat-based diets could further elaborate the

OPEN ACCESS

Edited by:

Dimitris G. Hatzinikolaou, National and Kapodistrian University of Athens, Greece

Reviewed by:

Giacomo Biagi,

University of Bologna, Italy

Bi E. Tan,

Hunan Agricultural University, China

${ }^{*}$ Correspondence: Yunhe Cao caoyh@cau.edu.cn Suozhu Liu

liusuozhu@xza.edu.cn

Specialty section: This article was submitted to Systems Microbiology, a section of the journal Frontiers in Microbiology

Received: 11 August 2021 Accepted: 21 September 2021

Published: 13 October 2021

Citation:

Wang J, Cao H, Bao C, Liu Y,

Dong $B$, Wang $C$, Shang $Z$, Cao $Y$ and Liu $S$ (2021) Effects of Xylanase in Corn- or Wheat-Based Diets on Cecal Microbiota of Broilers.

Front. Microbiol. 12:757066.

doi: 10.3389/fmicb.2021.757066 mechanism of the specificity of xylanase for different cereal grains. Thus, a total of 192 one-day-old broilers were randomly allotted into four dietary treatments, including wheatsoybean basal diet, wheat-soybean basal diet with 4,000 U/kg xylanase, corn-soybean basal diet, and corn-soybean basal diet with $4,000 \mathrm{U} / \mathrm{kg}$ xylanase to evaluate interactive effects of xylanase in corn- or wheat-based diets on broilers cecal microbiota during a 6 -week production period. The results indicated that bacterial community clustering was mainly due to cereal grains rather than xylanase supplementation. Compared with broilers fed wheat-based diets, corn-based diets increased alpha-diversity and separated from wheat-based diets $(p<0.05)$. Xylanase modulated the abundance of specific bacteria without changing overall microbial structure. In broilers fed wheat-based diets, xylanase increased the abundance of Lactobacillus, Bifidobacterium, and some butyrate-producing bacteria, and decreased the abundance of non-starch polysaccharides-degrading (NSP) bacteria, such as Ruminococcaceae and Bacteroidetes $(p<0.05)$. In broilers fed cornbased diets, xylanase decreased the abundance of harmful bacteria (such as genus Faecalitalea and Escherichia-Shigella) and promoted the abundance of beneficial bacteria (such as Anaerofustis and Lachnospiraceae_UCG_010) in the cecum $(p<0.05)$. Overall, xylanase supplementation to wheat- or corn-based diets improved broilers performance and cecal microbiota composition. Xylanase supplementation to wheat-based diets increased the abundance of butyrate-producing bacteria and decreased the abundance of NSP-degrading bacteria. Moreover, positive effects of xylanase on cecal microbiota of broilers fed corn-based diets were mostly related to the inhibition of potentially pathogenic bacteria, and xylanase supplementation to corn-based diets slightly affected the abundance of butyrate-producing bacteria and NSP-degrading bacterium, the difference might be related to lower content of $A X$ in corn compared to wheat.

Keywords: xylanase, wheat, corn, broiler, cecal microbiota 


\section{INTRODUCTION}

Xylanase has been widely applied in the broiler industry due to its ability to degrade arabinoxylans (AX) in poultry diets (Bautil et al., 2019). Corn and wheat are dominant feed grains for broilers diets; however, concentration and structure of $\mathrm{AX}$ in corn and wheat are different (Cowieson, 2010). At the present, most reports have focused on positive effects of xylanase on performance in broilers fed viscous wheat-based diets (Gonzalez-Ortiz et al., 2017; Liu and Kim, 2017; Wang et al., 2021). However, benefits of xylanase on growth performance of broilers supplemented non-viscous corn-based diets also were recorded (Khadem et al., 2016). Xylanase can reduce chyme viscosity, release nutrients from inside cell wall, and xylo-oligosaccharides (XOS) as a result of AX degradation in the gastrointestinal tract (GIT), which may then act like a prebiotic (Gonzalez-Ortiz et al., 2017; Petry et al., 2021). Previous studies have demonstrated that the dominant mode of action responsible for benefits of xylanase in corn- or wheatbased diets may be distinct and impacts of xylanase on growth performance, nutrient utilization, digesta characteristics, and cecal volatile fatty acid (VFA) concentrations of broilers fed corn- or wheat-based diets were compared (Kiarie et al., 2014; Munyaka et al., 2016; McCafferty et al., 2019a,b).

Cecal microbiota has the most complex microbiota in GIT which was associated with the health of broilers (Yadav and Jha, 2019). The GIT microbiota is shaped by both the environment and the broiler itself (Liu et al., 2021). Environmental changes, especially dietary factors, such as feed components and additives, have been widely demonstrated to regulate GIT microbiota (Shang et al., 2018). Cecal VFA concentrations are considered to be associated with bacterial fermentation in the large intestine (Ríos-Covián et al., 2016). Xylanase changed VFA concentrations in broilers cecum when the diets were corn- or wheat-based (McCafferty et al., 2019a,b). We hypothesized that supplemental xylanase might exert positive effects on cecal microbiota of broilers fed corn- or wheat-based diets by different gut bacterial communities.

16S rRNA gene sequencing analysis is a tool that has been widely applied to evaluate the diversity of GIT microbiota (Shang et al., 2018). To further elaborate the specificity of xylanase for feed ingredients, and the impacts of xylanase on cecal microbiota, we conducted the following experiment by feeding broilers corn- or wheat-based diets for a 6-week period and assessed the bacterial composition by $16 \mathrm{~S}$ rRNA gene sequencing.

\section{MATERIALS AND METHODS}

The present study was approved by the Laboratory Animal Welfare and Animal Experimental Ethical Inspection Committee of China Agricultural University (Beijing, China).

\section{Preparation of Xylanase}

The xylanase $(2,000,000 \mathrm{U} / \mathrm{kg})$ used in this experiment was preserved by our laboratory. An acidic xylanase gene $(x y n A)$ cloned from Aspergillus sulphureus was coden-optimized (GenBank accession number: EF114744) and expressed in Pichia pastoris (Cao et al., 2007). A disulfide bridge and proline residue substitutions were introduced to create xylanase mutants (AT-xynA) aiming to improve the thermostability of AT-xynA. Moreover, construction of a double-copy expression strain increased AT-xynA produced in Pichia pastoris (Wang et al., 2020). AT-xynA exhibited a resistance to an acidic environment (over $70 \%$ residual activity remained after $120 \mathrm{~min}$ incubation at $\mathrm{pH}$ 2.2) and was stable at high temperature (about $50 \%$ residual activity remained after $9.5 \mathrm{~min}$ incubation at $70^{\circ} \mathrm{C}$; Yang et al., 2017a). High-density fermentation of the xylanase was processed in a 15-L fermenter as previously described (Yang et al., 2017a). The actual xylanase product was obtained by mixing $66 \%$ of the liquid fermentation broth produced above with $34 \%$ soybean meal and then air dried for $24 \mathrm{~h}$. This resulted in a xylanase preparation containing approximately $2,000,000$ units $(\mathrm{U})$ of xylanase per $\mathrm{kg}$. One unit of xylanase activity is defined as the amount of enzyme releasing $1 \mu \mathrm{mol}$ of xylose per min under pH 3.0 and $50^{\circ} \mathrm{C}$ (Yang et al., 2017a).

\section{Experiment Animals, Management, Design, and Diets}

Individually numbered metal tags were attached to the wings of 192 1-day-old Arbor Acres broilers $(46.14 \pm 0.42 \mathrm{~g}$ ) for individual identification. Broilers were vaccinated with Newcastle disease vaccine on 7 and 28 , and vaccinated with an inactivated infectious bursal disease vaccine on $\mathrm{d} 14$ and 21 . The broilers were raised in an environmentally controlled room, and they were exposed to constant light. The initial room temperature for 1-day-old broilers was controlled at about $33^{\circ} \mathrm{C}$, and the temperature reduced $3^{\circ} \mathrm{C} /$ week until $24^{\circ} \mathrm{C}$ as the age of broilers increased. Broilers were randomly assigned to one of four diet treatments, and each treatment had six replicates with eight broilers. Broilers were fed an early phase diet (d 1 to 21 ) and a late phase diet (d 21 to 42). Dietary treatments consisted of $2 \times 2$ factorial design including two cereals (corn and wheat) and two enzyme treatments (control and xylanase): (1) wheatsoybean-based diet (WCON), (2) wheat-soybean-based diet with 4,000 U/kg xylanase (WXY), (3) corn-soybean-based diet (CCON), and (4) corn-soybean-based diet with 4,000 U/kg xylanase (CXY). The composition of experimental diets was shown (Table 1), and nutrient levels of diets satisfied National Research Council (National Research Council, 1994).

\section{Sample Collection and Processing}

Average daily gain (ADG), average daily feed intake (ADFI), and feed conversion ratio (FCR) of broilers from d 1 to 42 were calculated based on body weight and feed intake. One broiler from each replicate was slaughtered on d 14, 28, and 42 for collection of cecal digesta. The cecal digesta was snapfrozen in liquid nitrogen and then stored at $-80^{\circ} \mathrm{C}$.

\section{Cecal Microbiota Analysis}

For microbial diversity analysis, total genomic DNA from cecal digesta samples was extracted by using the EZNA stool 
TABLE 1 | Composition and nutrient levels of basal diets (\%, as fed basis).

\begin{tabular}{|c|c|c|c|c|}
\hline \multirow{2}{*}{ Items } & \multicolumn{2}{|c|}{1 to $21 \mathrm{~d}$ of age } & \multicolumn{2}{|c|}{22 to $42 \mathrm{~d}$ of age } \\
\hline & Corn & Wheat & Corn & Wheat \\
\hline \multicolumn{5}{|l|}{ Ingredients } \\
\hline Corn & 61.15 & - & 67.99 & - \\
\hline Wheat & - & 62.16 & - & 67.28 \\
\hline $\begin{array}{l}\text { Soybean meal, } 43 \% \text { crude } \\
\text { protein }\end{array}$ & 28.00 & 25.80 & 22.00 & 19.47 \\
\hline Fish meal, $64.6 \%$ crude protein & 3.71 & 3.46 & 2.60 & 4.62 \\
\hline Soybean oil & 3.00 & 4.96 & 3.60 & 5.56 \\
\hline Dicalcium phosphate & 1.28 & 1.02 & 1.38 & 0.83 \\
\hline Limestone & 1.26 & 1.40 & 1.10 & 1.16 \\
\hline Salt & 0.30 & 0.30 & 0.30 & 0.30 \\
\hline L-lysine $\mathrm{HCl}, 78 \%$ & - & - & 0.12 & - \\
\hline DL-Methionine, $98 \%$ & 0.15 & 0.14 & 0.07 & 0.02 \\
\hline L-Threonine, 98\% & 0.04 & 0.01 & 0.09 & 0.01 \\
\hline Chromic oxide & 0.25 & 0.25 & 0.25 & 0.25 \\
\hline$V$ itamin-mineral premix ${ }^{1}$ & 0.50 & 0.50 & 0.50 & 0.50 \\
\hline Total & 100.00 & 100.00 & 100.00 & 100.00 \\
\hline \multicolumn{5}{|l|}{ Calculated nutrient content ${ }^{2}$} \\
\hline Metabolizable energy, kcal/kg & 3,052 & 3,050 & 3,157 & 3,150 \\
\hline Crude protein & 20.08 & 20.95 & 19.37 & 20.36 \\
\hline Calcium & 1.00 & 1.00 & 0.90 & 0.90 \\
\hline Available phosphorous & 0.45 & 0.45 & 0.35 & 0.35 \\
\hline Digestible lysine & 0.86 & 0.86 & 0.73 & 0.73 \\
\hline Digestible methionine & 0.30 & 0.30 & 0.28 & 0.28 \\
\hline Digestible threonine & 0.63 & 0.63 & 0.56 & 0.56 \\
\hline
\end{tabular}

${ }^{1}$ Vitamin A, 11,000IU; vitamin D, 3,025IU; vitamin E, $22 \mathrm{mg}$; vitamin $K_{3}, 2.2 \mathrm{mg}$; thiamine, 1.65 mg; riboflavin, 6.6mg; pyridoxine, 3.3mg; cobalamin, $17.6 \mu$; nicotinic acid, $22 \mathrm{mg}$; pantothenic acid, $13.2 \mathrm{mg}$; folic acid, $0.33 \mathrm{mg}$; biotin, $88 \mu \mathrm{g}$; choline chloride, 500 mg; iron, 48mg; zinc, 96.6mg; manganese, 101.76 mg; copper, 10mg; selenium, $0.05 \mathrm{mg}$; iodine, $0.96 \mathrm{mg}$; and cobalt, $0.3 \mathrm{mg}$.

${ }^{2}$ Values were calculated according to the National Research Council (1994).

DNA kit (Omega Biotek, Norcross, GA). The concentration of DNA was quantified by a NanoDrop 2000 spectrophotometer (Thermo Scientific, MA, United States), and the quality of DNA was analyzed by electrophoresis with $2 \%$ agarose gels. Then, the V3-V4 region of the 16S rRNA gene was amplified using genomic DNA of cecal digest samples as the template, and 338F (5'barcodeACTCCTACGGGAGGCAGCAG3') and 806R (5'GGACTACHVGGGTW TCTAAT3') as the primers. The PCR reaction conditions were as: initial denaturation at $95^{\circ} \mathrm{C}$ for $3 \mathrm{~min}$, then $27 \mathrm{cycles}$ of $95^{\circ} \mathrm{C}$ for $30 \mathrm{~s}, 55^{\circ} \mathrm{C}$ for $30 \mathrm{~s}$ and $72^{\circ} \mathrm{C}$ for $45 \mathrm{~s}$, and finally $72^{\circ} \mathrm{C}$ for $5 \mathrm{~min}$. The PCR products were purified by the AxyPrep DNA Gel Extraction Kit (Axygen Biosciences, Union City, CA). The purified amplicons were pooled in equimolar concentrations and then sequenced on Illumina MiSeq platform. Trimmomatic (version 3.29) was applied to quality-filter raw Illumina fastq files. Operational taxonomic units (OTUs) were clustered with a similarity of $97 \%$ using UPARSE (version 7.0). ${ }^{1}$ The raw data have been submitted to the NCBI Sequence Read Archive (SRA) database (PRJNA753623).

${ }^{1}$ http://drive5.com/uparse/

\section{Statistical Measurements}

To determine interaction effects of cereal type and xylanase supplementation on growth performance and microbiota alphadiversity of broilers, a two-way ANOVA was performed using SAS (version 9.2, 2008). Each treatment contained six pens, and the pen was considered as an experiment unit. $p<0.05$ was considered significant, and $0.05 \leq p \leq 0.10$ was regarded a trend. When a significant effect was observed, the data were further analyzed by one-way ANOVA (version 9.2, 2008). The rarefaction curves of OTUs were calculated by QIIME to determine the sequencing coverage across samples. Alphadiversity was calculated by Sobs (the observed OTUs), Chao (the Chao 1 estimator), Ace (the ACE estimator), and PD (Phylogenetic diversity). Principal coordinate analysis (PCoA) on Weighted UniFrac distance was conducted. Analysis of similarity (ANOSIM) with 999 permutations was used to assess the similarity between bacterial community structure by measuring $R$ value (between 0 and 1). $R=0$ indicates that two groups are similar, and $R=1$ suggests two groups are different. The linear discriminant analysis effect size (LEfSe) method was applied for the analysis of significant differences in abundance of microbiota community.

\section{RESULTS}

\section{Growth Performance}

The ADG, ADFI, and FCR of broilers from different dietary treatments were shown in Figure 1. From 1 to $42 \mathrm{~d}$ of age, ADG and ADFI of broilers from WXY and CCON groups were higher than broilers from WCON group $(p<0.05)$; however, ADG and ADFI of broilers from CXY group were higher compared with broilers from WXY to CCON groups $(p<0.05)$. Broilers from WCON group exhibited higher FCR compared to broilers from WXY and CXY groups $(p<0.05)$, and FCR of broilers from CCON group was similar to those from WCON, WXY, and CXY groups ( $p>0.05)$. Additionally, cereal main effect influenced ADG and ADFI $(p<0.05)$, the broilers receiving corn-based diets showed higher ADG and ADFI than those fed wheat-based diets $(p<0.05)$. Xylanase main effect also influenced ADG and ADFI $(p<0.05)$, and tended to influence FCR $(p=0.05)$, xylanase supplementation to broilers diets could improve ADG, ADFI, and FCR of broilers $(p<0.05)$. However, no interactive effects of cereal type and xylanase were observed on broilers growth performance.

\section{Cecal Microbiota Analysis}

In this experiment, a total of 3,211,886 high-quality sequence reads of samples, with the average read length of 412 base (bp), were obtained for subsequent analysis. In total, 1,146 distinct OTUs with a $97 \%$ identity were generated that fell into 13 phyla, 24 classes, 44 orders, 70 families, and 175 genera. Rarefaction curves based on the observed OTUs approached asymptotic for each dietary treatment on $\mathrm{d} 14,28$, and 42 , indicating the availability of sufficient numbers of OTUs to represent each microbiota community, and age-linked increasing 

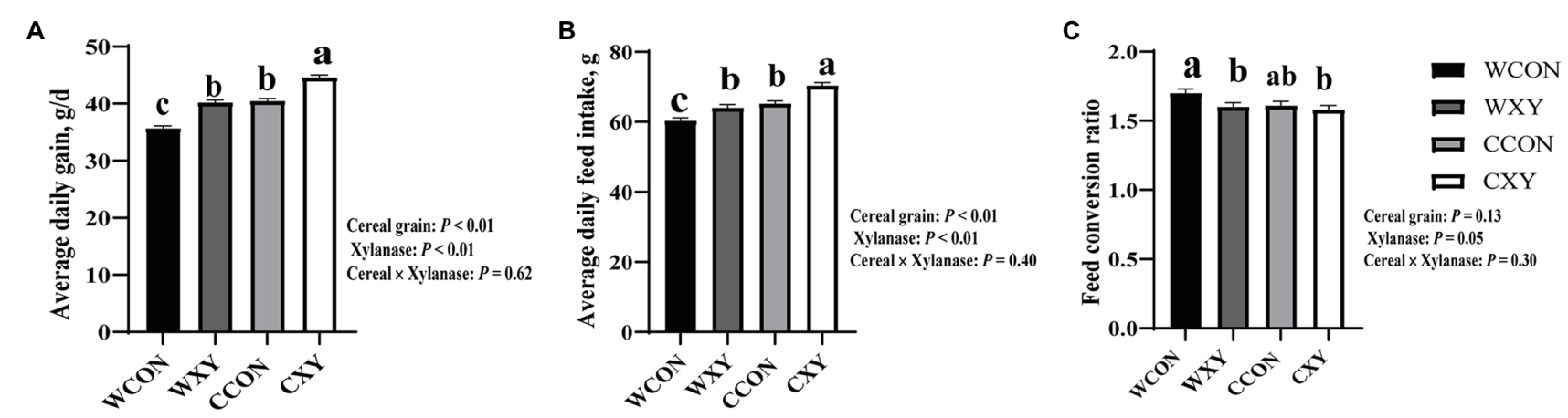

FIGURE 1 | Growth performance of broilers fed corn- or wheat-based diets with or without xylanase during 6-week period. Dietary treatments consisted of wheatsoybean basal diet (WCON), wheat-soybean basal diet with 4,000 U/kg xylanase (WXY), corn-soybean basal diet (CCON), and corn-soybean basal diet with $4,000 \mathrm{U} / \mathrm{kg}$ xylanase (CXY). (A) Average daily gain. (B) Average daily feed intake. (C) Feed conversion ratio. Significant differences $(p<0.05)$ among different groups are indicated by different letters. Values are expressed as means $\pm \mathrm{SEM}$.

trend in the number of observed OTUs was observed in broilers (Figure 2).

Venn diagrams were generated to make qualitative comparisons of the core and unique genera between WCON, WXY, CCON, and CXY groups (Figure 3). The core OTUs of the four treatments were 737 , and the number of unique genera for WCON, WXY, CCON, and CXY groups was 25, 29, 34, and 29, respectively. As shown in Table 2, there was not a significant interaction between cereal type and xylanase supplementation in cecal microbiota alpha-diversity of broilers $(p>0.05)$. On d 14, cereal main effect influenced indices of Sobs, Ace, Chao $(p<0.05)$, and PD $(p=0.08)$, and the broilers fed corn-based diets exhibited higher Sobs, Ace, Chao, and PD values compared to those receiving wheat-based diets $(p<0.05)$. On d $42, \mathrm{PD}$ values were also significantly affected by the cereal type $(p<0.05)$, and broilers from CXY exhibited significantly higher PD values compared with broilers from WCON to WXY groups $(p<0.05)$. The PCoA was performed by using Weighted UniFrac distance to determine $\beta$-diversity of microbial communities between different groups (Figure 4). Although the addition of xylanase in wheat- or corn-based diets improved growth performance of broilers, no distinct clustering due to xylanase supplementation was denoted. However, PCoA of the OTU data for chickens fed wheat- or corn-based diets revealed a less pronounced but distinct variation between cereal grains (ANOSIM, $R=0.1572, p=0.003$ ).

To further elaborate effects of xylanase supplementation to wheat- or corn-basal diets on broilers cecal microbiota, we then compared the bacterial community at phylum and family levels across four dietary treatments at each sampling time point. At the phylum level (Figure 5A), microbiota of cecal microbiota community was dominated by Firmicutes, Bacteroidetes, Proteobacteria, Tenericutes, and Actinobacteria independent of sampling time point and xylanase supplementation. Two major phyla (Firmicutes and Bacteroidetes) accounted for more than $90 \%$ of the cecal bacterial community at each time point in four treatments. The other phyla, such as Proteobacteria, Tenericutes, and Actinobacteria, were represented at relatively low abundance. Bacteroidetes increased in abundance with time in four dietary treatments; however, the relative abundance of Firmicutes decreased with age. At the family level (Figure 5B), Ruminococcaceae, Lachnospiraceae, Clostridiales_vadinBB60_ group, Lactobacillaceae, Peptococcaceae, and Erysipelotrichaceae assigned to Firmicutes phyla, Porphyromonadaceae, Rikenellaceae, and Bacteroidaceae assigned to Bacteroidetes phyla, and norank_o__Mollicutes_RF9 which belongs to Tenericutes phyla were dominant families in the cecum. At 14 days of age, Ruminococcaceae was the most abundant family in WCON, CCON, and CXY groups, and Lachnospiraceae was the most abundant family in WXY group. At 28 days of age, Ruminococcaceae was the most abundant family, and at 42 days of age, Porphyromonadaceae was the dominant family in four dietary treatments.

To further probe how AT-xynA modulated GIT microbial community, alterations in cecal microbiota were also analyzed by the LefSe method on d 14, 28, and 42. On d 14 (Figure 6A), in WCON and WXY groups, the abundance of genus norank $f_{-}$ Lachnospiraceae, Shuttleworthia, Ruminococcus_torques_group, and Sellimonas (family Lachnospiraceae) was higher in WXY group $(p<0.05)$. The abundance of genus Ruminococcaceae $U C G \_004$, unclassified_f_Ruminococcaceae, Anaerotruncus, and Butyricicoccus (family Ruminococcaceae) was also higher in WXY group, while the abundance of family Ruminococcaceae was higher in WCON group $(p<0.05)$. The relative abundance of genus Defluviitaleaceae_UCG_011 (family Defluviitaleaceae), Clostridium_sensu_stricto_1, Anaerofustis (family Eubacteriaceae), and norank_f_Erysipelotrichaceae was higher in WXY group $(p<0.05)$. The phylum Actinobacteria, order Coriobacteriales, and genus Bifidobacterium, Gordonibacter, and Senegalimassilia were also higher in WXY group ( $p<0.05)$. On d 14 (Figure 7A), in CCON and CXY groups, higher relative abundance of Ruminococcus_gauvreauii_group, Eubacterium_brachy_group, and Family_XIII_UCG_001 was observed in CXY group $(p<0.05)$. The abundance of genus Lachnospiraceae_FCS020_group, Faecalitalea, Eubacterium_hallii_group, Flavonifractor, Intestinimonas, Candidatus_Soleaferrea, and Ruminococcaceae_ $N K 4 A 214$ group was higher in CCON group $(p<0.05)$. The abundance of genus Christensenellaceae_R_7_group (family 


\section{Rarefaction curves}

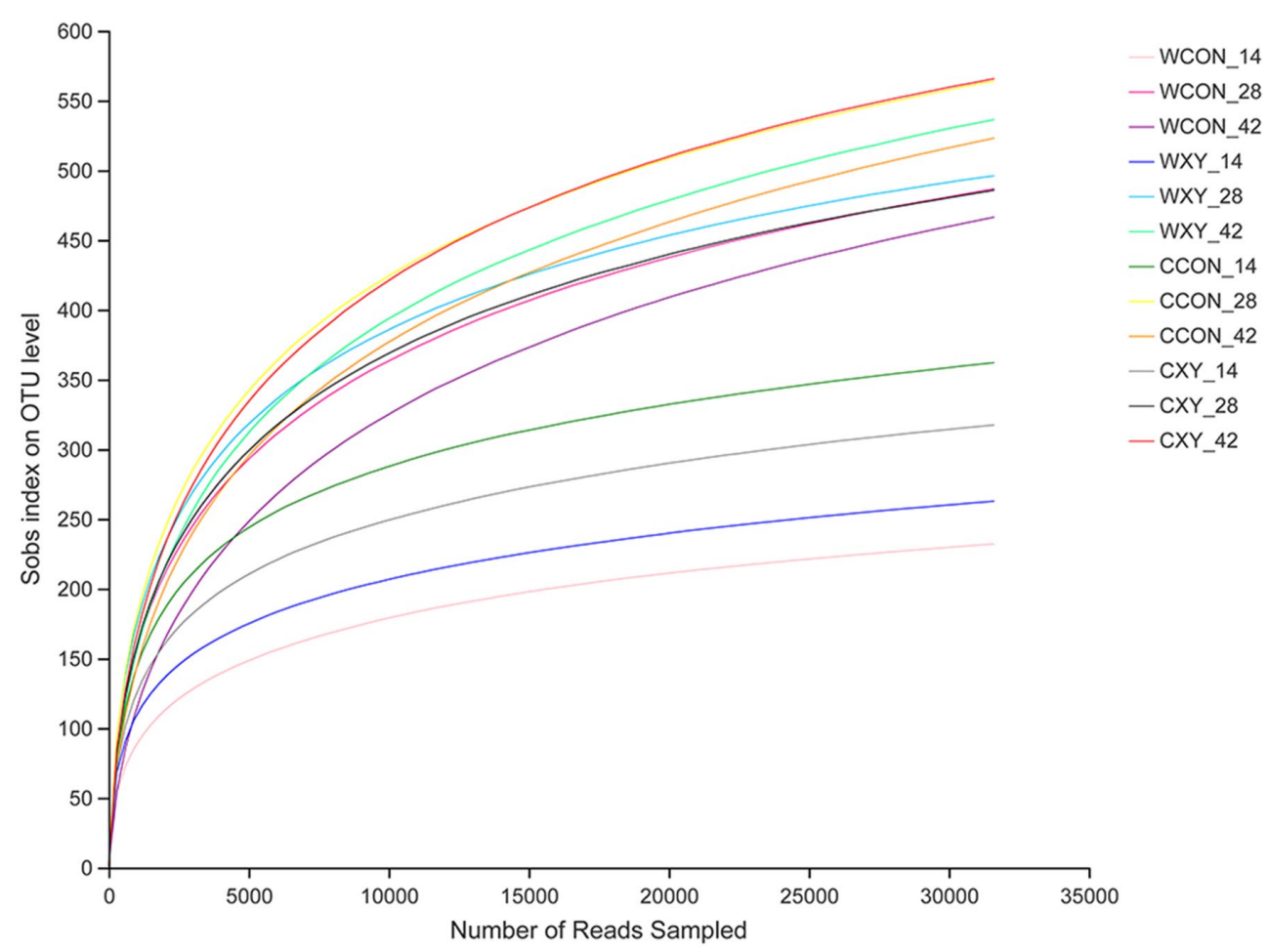

FIGURE 2 | Rarefaction curves showing sequence coverage across samples on the observed number of OTUs. For rarefaction plots, calculated based on the observed OTUs to determine the sequencing coverage across samples. OTUs, operational taxonomic units. Wheat-soybean basal diet (WCON); wheat-soybean basal diet with 4,000 U/kg xylanase (WXY); corn-soybean basal diet (CCON); and corn-soybean basal diet with 4,000 U/kg xylanase (CXY).

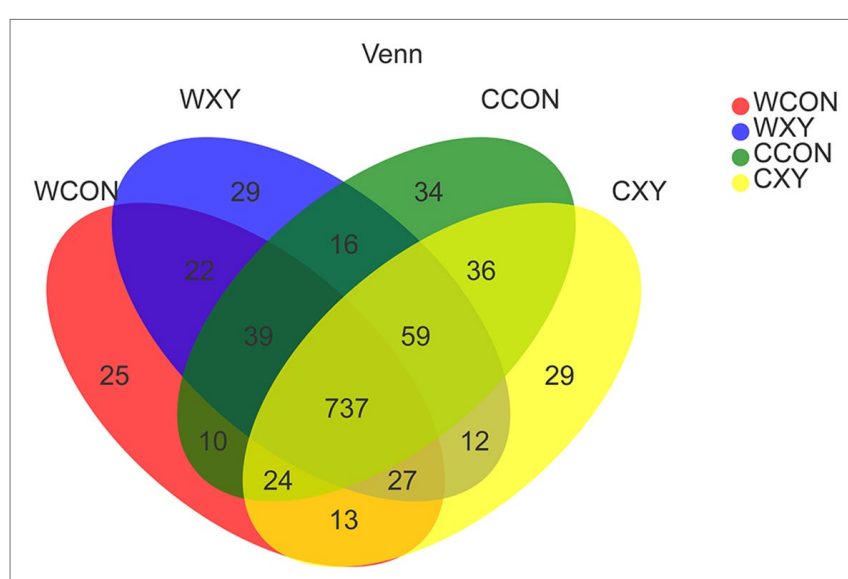

FIGURE 3 | Bacterial OTU community composition of the broilers fed corn- or wheat-based diets. Venn diagrams were generated to make qualitative comparisons of the core and unique OTUs among WCON, WXY, CCON, and CXY groups. OTUs, operational taxonomic units. Wheat-soybean basal diet (WCON); wheat-soybean basal diet with 4,000U/kg xylanase (WXY); corn-soybean basal diet (CCON); and corn-soybean basal diet with 4,000U/kg xylanase (CXY).

Christensenellaceae), Defluviitaleaceae_UCG_011 (family Defluviitaleaceae), unclassified_f__Bacillaceae (order Bacillales), and norank_o_Mollicutes_RF9 (order Mollicutes_RF9) was increased in CCON group $(p<0.05)$. The abundance of phylum Proteobacteria including family Enterobacteriaceae and family Desulfovibrionaceae was also higher in CCON group $(p<0.05)$.

On d 28 (Figure 6B), in WCON and WXY groups, the phylum Firmicutes were higher in WXY group, while the phylum Bacteroidetes were higher in WCON group $(p<0.05)$. The genus Anaeroplasma belonged to order Anaeroplasmatales and the genus Odoribacter, Hydrogenoanaerobacterium, and Ruminococcaceae_V9D2013_group were higher in WXY group $(p<0.05)$. On d 28 (Figure 7B), in CCON and CXY groups, the abundance of class Clostridia including genus Anaerotruncus, Tyzzerella, and Caproiciproducens, as well as the phylum Lentisphaerae, was higher in CXY group $(p<0.05)$. The abundance of genus Alistipes, Eubacterium_ventriosum_group, norank_f Peptococcaceae, Senegalimassilia, and norank_f_LLachnospiraceae was higher in CCON group $(p<0.05)$.

On d 42 (Figure 6C), in WCON and WXY groups, the abundance of genus Eubacterium_hallii_group, Eubacterium_ ventriosum_group, Gordonibacter, and Parabacteroides was higher in WXY group $(p<0.05)$. The abundance of genus Anaerotruncus, unclassified_f_Ruminococcaceae, Subdoligranulum, and Caproiciproducens (family Ruminococcaceae) was also higher in WXY group $(p<0.05)$. Higher abundance of the genus Clostridium sensu_stricto_1 belonging to family Clostridiaceae_1, genus Defluviitaleaceae_UCG_011 belonging to family Defluviitaleaceae, 
TABLE 2 | Effects of xylanase on alpha-diversity of cecal bacterial community in broilers fed wheat- or corn-based diets'.

\begin{tabular}{|c|c|c|c|c|c|c|c|c|}
\hline & WCON & WXY & CCON & CXY & SEM & \multicolumn{3}{|c|}{ Analysis of Variance } \\
\hline \multicolumn{9}{|l|}{ d 14} \\
\hline $\begin{array}{l}\text { The observed OTUs } \\
\text { The ACE estimator } \\
\text { The Chao }{ }^{1} \text { estimator } \\
\text { Phylogenetic diversity }\end{array}$ & $\begin{array}{c}232.00^{c} \\
271.49^{b} \\
276.91^{b} \\
17.63\end{array}$ & $\begin{array}{c}262.67^{\mathrm{bc}} \\
311.52^{\mathrm{ab}} \\
315.58^{\mathrm{b}} \\
22.61\end{array}$ & $\begin{array}{c}362.00^{\mathrm{a}} \\
420.13^{\mathrm{a}} \\
422.40^{\mathrm{a}} \\
28.00\end{array}$ & $\begin{array}{c}317.33^{\mathrm{ab}} \\
368.80^{\mathrm{ab}} \\
374.52^{\mathrm{ab}} \\
24.90\end{array}$ & $\begin{array}{r}24.46 \\
32.44 \\
34.35 \\
3.15\end{array}$ & $\begin{array}{l}0.16 \\
0.20 \\
0.16 \\
0.24\end{array}$ & $\begin{array}{r}<0.01 \\
0.01 \\
0.01 \\
0.08\end{array}$ & $\begin{array}{l}0.78 \\
0.87 \\
0.68 \\
0.77\end{array}$ \\
\hline \multicolumn{9}{|l|}{ d 28} \\
\hline $\begin{array}{l}\text { The observed OTUs } \\
\text { The ACE estimator } \\
\text { The Chao }{ }^{1} \text { estimator } \\
\text { Phylogenetic diversity }\end{array}$ & $\begin{array}{r}486.67 \\
579.62 \\
581.69 \\
32.74\end{array}$ & $\begin{array}{r}496.00 \\
567.51 \\
564.43 \\
32.99\end{array}$ & $\begin{array}{r}564.00 \\
667.31 \\
686.76 \\
35.40\end{array}$ & $\begin{array}{r}485.67 \\
569.31 \\
570.28 \\
32.96\end{array}$ & $\begin{array}{r}35.22 \\
29.94 \\
34.81 \\
1.92\end{array}$ & $\begin{array}{l}0.25 \\
0.19 \\
0.19 \\
0.50\end{array}$ & $\begin{array}{l}0.37 \\
0.17 \\
0.15 \\
0.51\end{array}$ & $\begin{array}{l}0.36 \\
0.10 \\
0.10 \\
0.58\end{array}$ \\
\hline \multicolumn{9}{|l|}{ d 42} \\
\hline
\end{tabular}

${ }^{1}$ Alpha diversity analysis for bacterial community determined by $16 \mathrm{~s}$ RNA sequencing. OTUs, operational taxonomic units. Wheat-soybean basal diet (WCON); wheat-soybean basal diet with 4,000 U/kg xylanase (WXY); corn-soybean basal diet (CCON); and corn-soybean basal diet with 4,000 U/kg xylanase (CXY).

${ }^{a-c}$ Means with a row with no common superscript differ significantly.

and genus Lactobacillus from class Bacilli was observed in WXY group $(p<0.05)$. On d 42 (Figure 7C), in CCON and CXY groups, the abundance of class Erysipelotrichia, genus Lachnospiraceae UCG_010, Subdoligranulum, Anaerofustis, and norank $f_{-}$ Runmiococcaceae was higher in CXY group, while the abundance of genus Ruminiclostridium_1, Senegalimassilia, Flavonifractor, and norank_f_Erysipelotrichaceae was higher in CCON group $(p<0.05)$.

The cecal microbiota of broilers fed corn- or wheat-based diets was also compared by the LefSe method (Figure 8). The abundance of genus Lachnospiraceae_UCG_010, Tyzzerella, Shuttleworthia, Eubacterium_ventriosum_group, Ruminococcaceae_UCG_013, Ruminococcaceae_UCG_004, Oscillospira, Anaerofilum, Butyricicoccus, Ruminococcaceae UCG_008, Ruminococcaceae_UCG_005, Intestinimonas, Ruminococcus_2, norank_f__Ruminococcaceae, Oscillibacter, and Ruminiclostridium_9 was higher in broilers fed cornbased diets $(p<0.05)$. The abundance of genus Christensenellaceae_R_7_group (Christensenellaceae family), Romboutsia (Peptostreptococcaceae family), genus unclassified_f__Propionibacteriaceae (Peptococcaceae family), Erysipelatoclostridium, Turicibacter, Dielma, Erysipelotrichaceae_UCG_003, Faecalitalea (family Erysipelotrichaceae), Elusimicrobium (phylum Elusimicrobia), unclassified_f__Peptococcaceae (family Propionibacteriaceae), Eggerthella, Gordonibacter (family Coriobacteriaceae), Methylomicrobium (family Methylococcaceae), and Methylophilus (family Methylophilaceae) was higher in broilers receiving corn-based diets $(p<0.05)$. Moreover, higher abundance of the phylum Proteobacteria, order Bifidobacteriales, family Bifidobacteriaceae, genus unclassified_f__Lachnospiraceae, Marvinbryantia, Bifidobacterium, and Lactococcus was observed in broilers receiving wheat-based diets $(p<0.05)$.

\section{DISCUSSION}

In the current study, broilers receiving corn-based diets showed higher ADG and ADFI than broilers supplemented wheat-based diets. Masey-O'Neill et al. (2014) also reported that feeding broilers with corn-based diets increased body weight gain and feed intake compared to broilers fed wheat-based diets from $\mathrm{d} 1$ to 28 . This observation might be related to the higher concentration of soluble NSP in viscous wheat. The soluble NSP is known to exhibit antinutritive effects by increasing intestinal digesta viscosity, affecting gut transit time, thus impair nutrient absorption and utilization of broilers (Kiarie et al., 2014). However, similar to our results, it has been reported that feeding broilers with wheatbased diets supplemented with xylanase could improve growth performance, the improvement might be associated with the alleviation of detrimental effects of soluble NSP on digesta viscosity (Gonzalez-Ortiz et al., 2017; Liu and Kim, 2017). Moreover, in this study, xylanase improved growth performance of broilers receiving corn-based diets. Benefits of xylanase on performance of broilers fed non-viscous corn-based diets also have been reported, indicating that there are other mechanisms besides this mode of viscosity reduction (Khadem et al., 2016). The primary mode of action may depend on the type of cereal grain, thus 16S rRNA gene high-throughput sequencing in this study was used to elaborate effects of xylanase on cecal microbiota of broilers supplemented corn- or wheat-based diets on d 14, 28, and 42 .

The generated rarefaction curves have reached plateau with the Sobs index, suggesting that $16 \mathrm{~S}$ rRNA sequencing libraries could represent most of OTUs present in all the samples sequenced in this study. As shown in Venn diagram, the number of OTUs in cecal microbiota among four dietary treatments was similar, and most of them shared. When comparing effects of xylanase on cecal microbiota alpha-diversity of broilers fed 

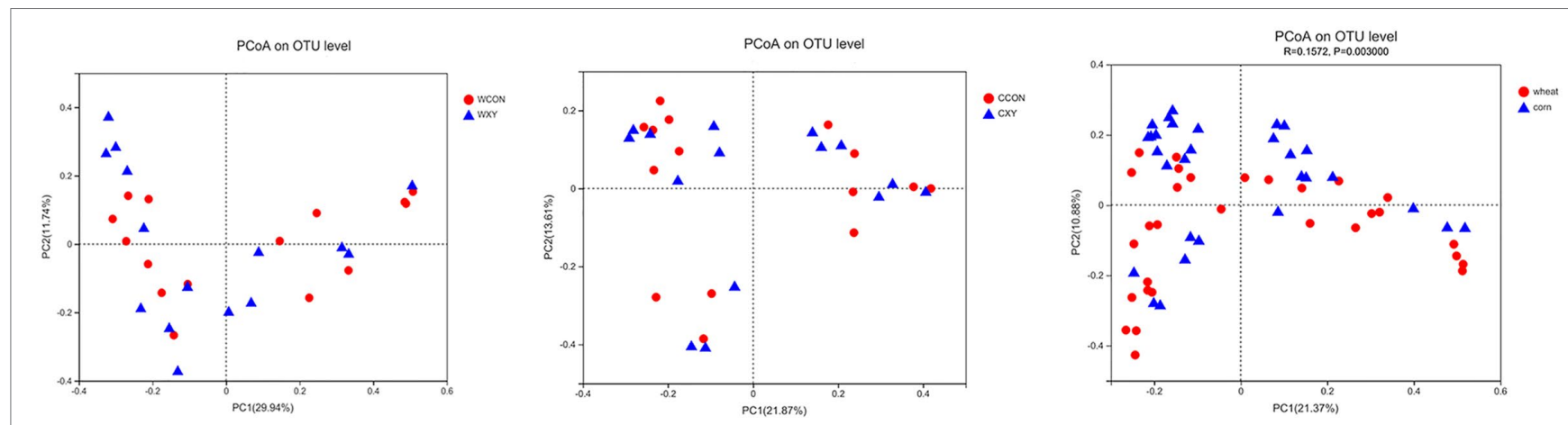

FIGURE 4 | Principal coordinate analysis (PCoA) representing similarity of cecal microbiota in broilers fed corn- or wheat-based diets. The PCoA was performed by using Weighted UniFrac distance to determine $\beta$-diversity of bacterial communities between different groups. No distinct clustering due to xylanase supplementation was denoted (WCON vs. WXY; CCON vs. CXY). However, a less pronounced but distinct variation was observed in broilers fed wheat- or corn-based diets (ANOSIM, $R=0.1572, P=0.003)$. Wheat-soybean basal diet (WCON); wheat-soybean basal diet with 4,000 U/kg xylanase (WXY); corn-soybean basal diet (CCON); and corn-soybean basal diet with 4,000 U/kg xylanase (CXY).

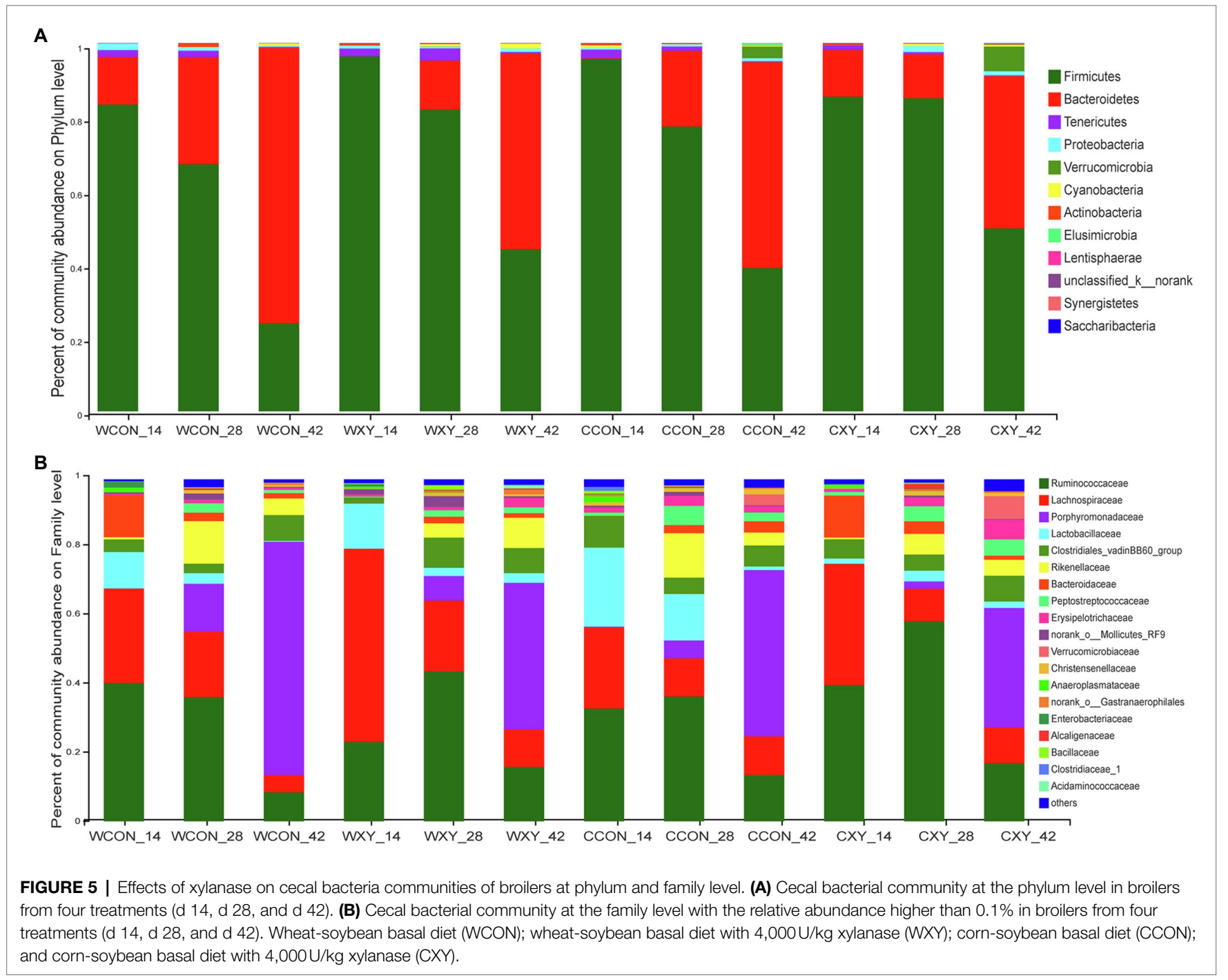

wheat- or corn-based diets, there was not a significant interaction between cereal type and xylanase supplementation in alphadiversity, and xylanase main effect also did not affect alpha-diversity of broilers. However, we found that broilers receiving corn-based diets had richer and more diverse microbiota than broilers fed wheat-based diets on d 14 and 42, indicating 
A

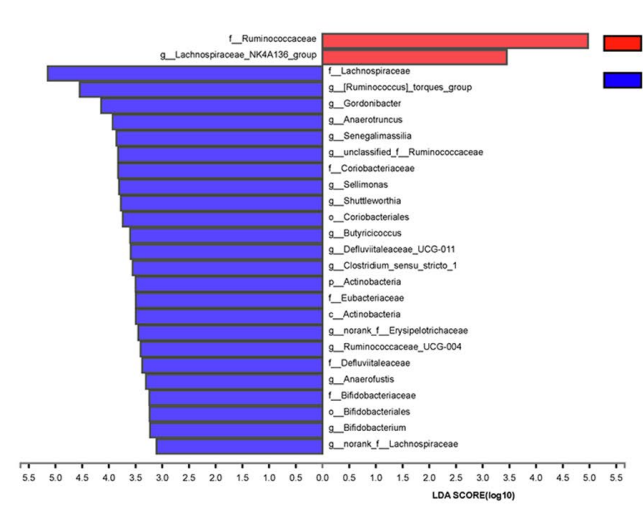

B

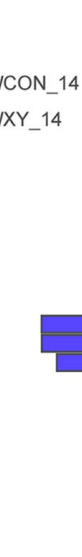

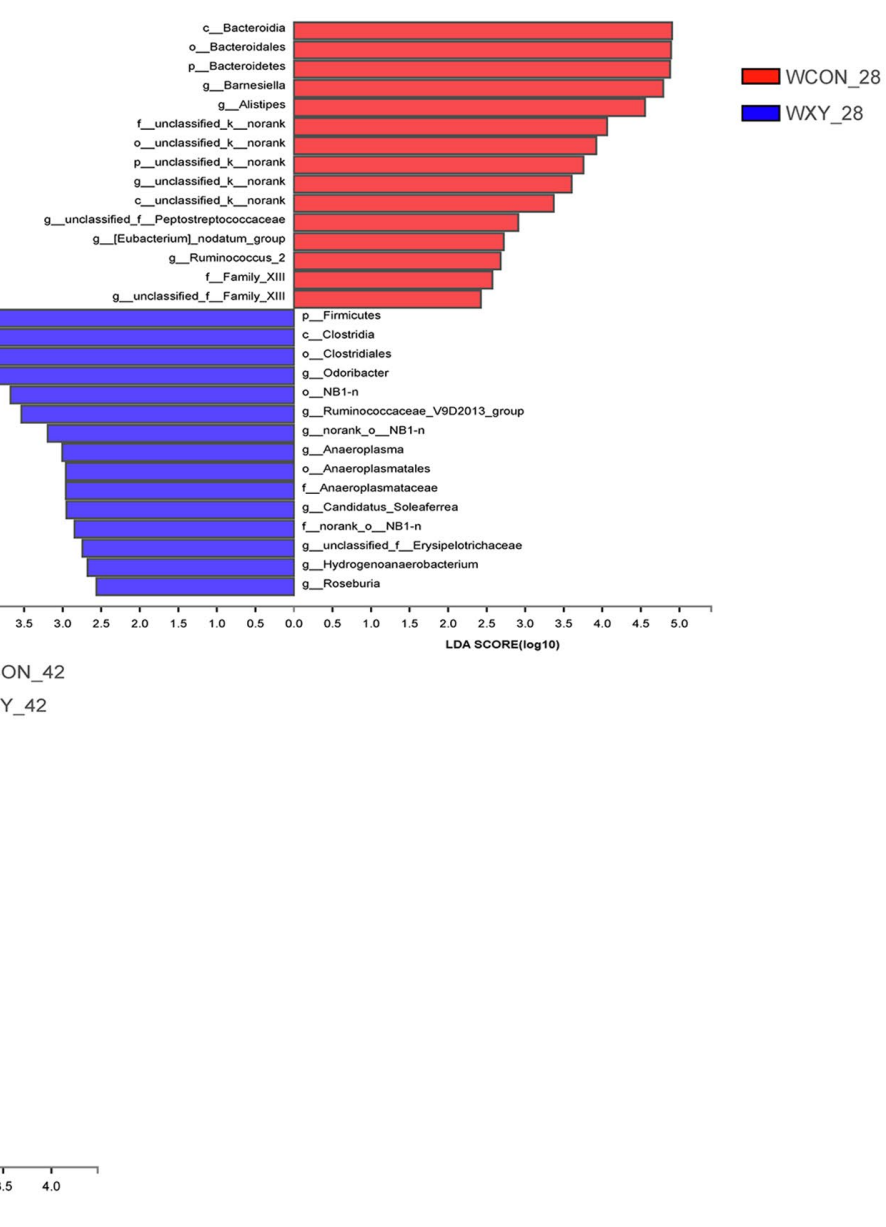

C

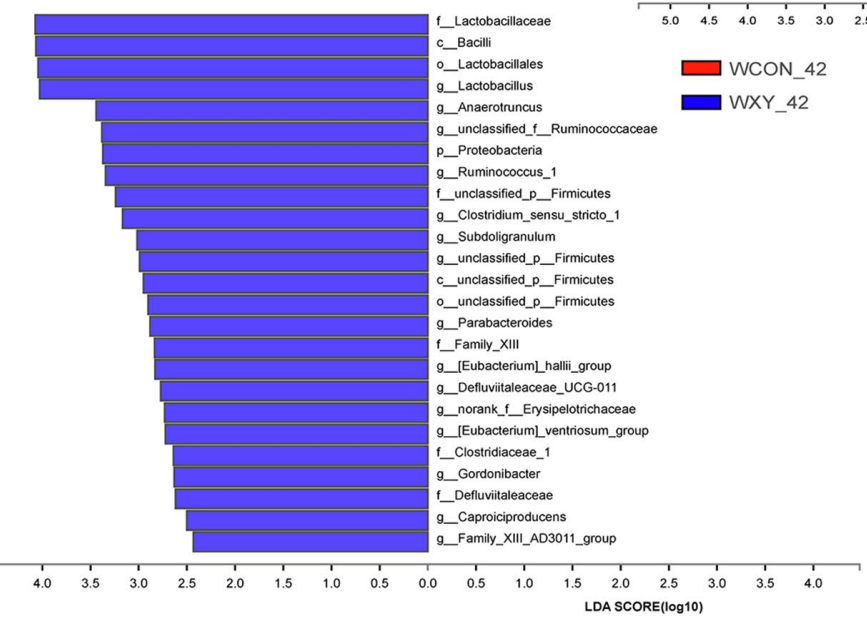

FIGURE 6 | The LEfSe analysis for cecal bacteria communities from phylum to genus level in WCON and WXY groups. Different bacterial communities from phylum to genus level between WCON (denoted by red bars) and WXY (denoted by blue bars) groups on d 14 (A), d 28 (B), and d 42 (C). Wheat-soybean basal diet (WCON); wheat-soybean basal diet with $4,000 \mathrm{U} / \mathrm{kg}$ xylanase (WXY).

that microbiota of broilers receiving corn-based diets exhibited higher richness. The increase in alpha-diversity has been reported to be beneficial to the health of broilers, the increased diversity might be able to promote the stability of microbiota (Díaz Carrasco et al., 2018; Dhillon et al., 2019). The analysis of PCoA based on dietary treatments did not exhibit distinct clustering patterns, the composition of GIT microbiota between WCON and WXY groups was similar, and beta diversity was also not different between CCON and CXY groups. However, the phylogenetic distances clearly revealed that type of cereal grain affected beta diversity, suggesting a distinction in microbial community structure of broilers receiving corn-based diets relative to broilers receiving wheat-based diets.

To further probe how xylanase modulates GIT microbial community of broilers receiving wheat- or corn-based diets, the variations in relative abundance of microbiota were analyzed. The development of GIT microbiota in broilers might be a continuous process of microbial community succession. In the current study, the development of microbiota community from an immature phase to a mature phase in four dietary treatments was clearly shown. As previously reported, cecal communities for all the broilers were dominated by members of Firmicutes and Bacteroidetes phylum (Ilaria et al., 2020). Firmicutes accounted for most of the cecal microbiota in young broilers, while Bacteroidetes were only enriched at the end of productive life. Butyrate-producing bacteria mostly belong to Firmicutes phylum (Chakraborti, 2015; Dai et al., 2021). Representatives of Bacteroidetes phylum mainly degrade polysaccharides producing propionate (Yang et al., 2013; Polansky et al., 2016). In quickly growing, young birds, butyrate is highly required to provide energy for the growth of intestinal cells, while adult birds have fully developed intestines and need less amount of butyrate (Petra et al., 2014; Onrust et al., 2015). Higher abundance of Bacteroidetes in adult broilers represented an effective balance between energy obtained from available nutrients and sustained growth (Ocejo et al., 2019). The alteration of the proportion of Firmicutes and Bacteroidetes indicates the change of produced butyrate in broilers cecum, thus can explain the results in this study that Bacteroidetes gradually increased throughout the production period. 


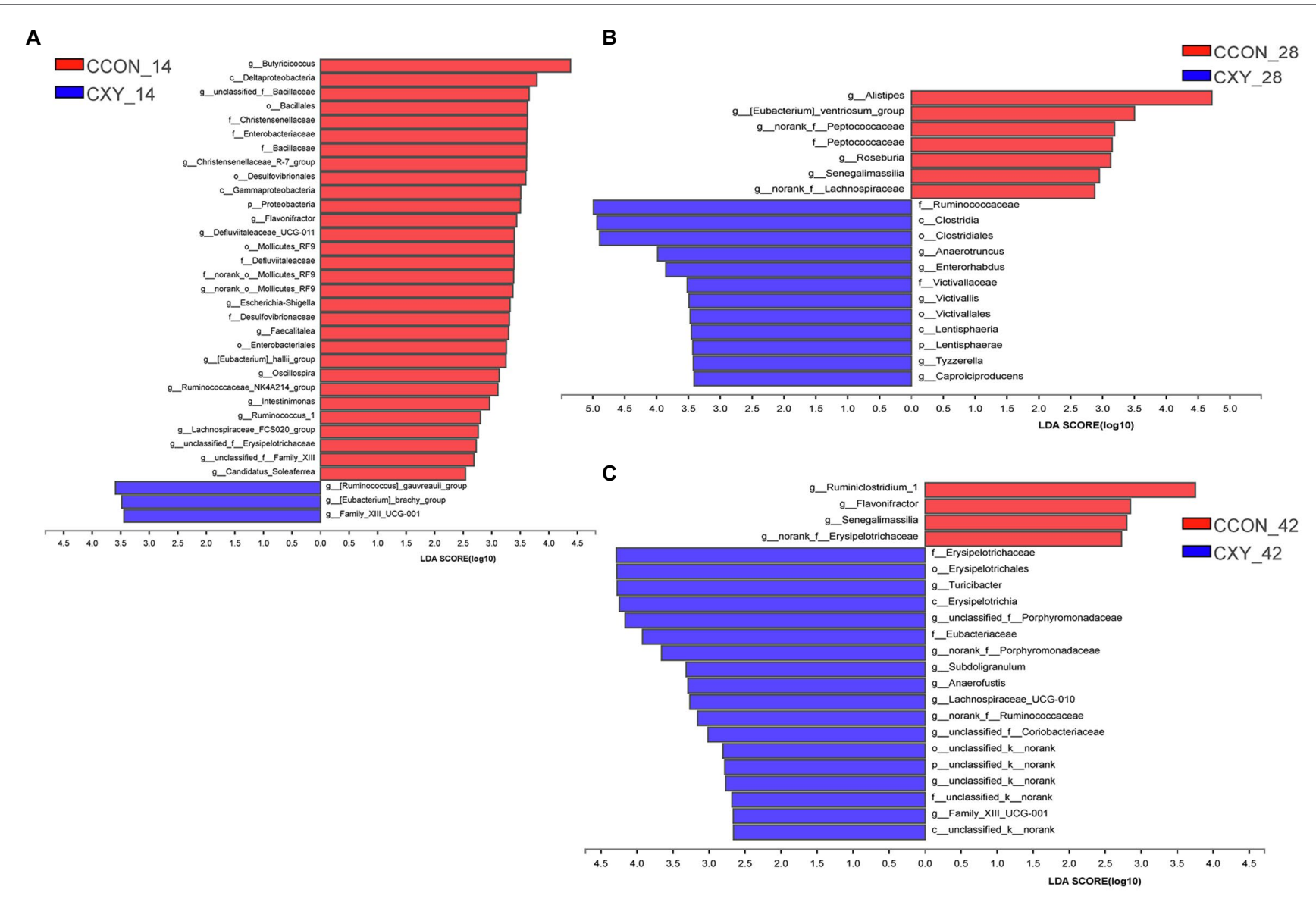

FIGURE 7 | The LEfSe analysis for cecal bacteria communities from phylum to genus level in CCON and CXY groups. Different bacterial communities from phylum to genus level between CCON (denoted by red bars) and CXY (denoted by blue bars) groups on d 14 (A), d 28 (B), and d 42 (C). Corn-soybean basal diet (CCON); corn-soybean basal diet with 4,000 U/kg xylanase (CXY).

When comparing cecal microbiota of broilers fed wheatbased diets, compared with WXY, higher abundance of family Ruminococcaceae on d 14 and phylum Bacteroidetes on d 28 was observed in WCON group. Ruminococcaceae has been reported to be related to cellulose-degrading capacity, and Ruminococcaceae is enriched in genes encoding for endo-1,4beta-xylanase and cellulase (Biddle et al., 2013; Dai et al., 2020). Phylum Bacteroidetes also contain a high number of genes encoding for glycose hydrolases, such as $\beta$-xylosidase, endo- $1,4-\beta$-xylanase, and $\alpha$ - $\mathrm{N}$-arabinofuranosidase, which makes Bacteroidetes become the primary phylum for the degradation of complex polysaccharides in GIT (Zhang et al., 2018a). Furthermore, it has been demonstrated that Bacteroides of the phylum Bacteroidetes was related to AX degradation (Zhang et al., 2018a,b). Dietary supplementation of xylanase to broilers could effectively hydrolyze AX in small intestines, thereby reducing the availability of polysaccharides to Ruminococcaceae and Bacteroidetes in the cecum. However, the abundance of microbiota associated with arabinoxylan-degrading capacity was not significantly different between broilers from CCON to CXY groups, we speculated that the difference might be related to the lower contents of arabinoxylans in corn.
Additionally, compared with WXY, the increased abundance of Ruminococcaceae in broilers from WCON group on $\mathrm{d}$ 14, and compared with CXY, higher abundance of order Mollicutes_RF9 in CCON group on d 14 was observed. Ruminococcaceae has been demonstrated to be sensitive to starch, increased abundance of Ruminococcaceae in broilers from WCON group might also be related to lower ileal starch digestibility, because the addition of xylanase has been reported to increase starch digestibility (Choct et al., 1999; McCafferty et al., 2019a). The metagenome-assembled genomes of Mollicutes_RF9 group also have the CAZyme related with starch degradation, thus lower concentration of undigested starch flowing into the cecum might limit the growth of Mollicutes_RF9 (Ma et al., 2019).

Compared to WCON, higher abundance of family Lachnospiraceae (containing norank_f_Lachnospiraceae, Shuttleworthia, Ruminococcus_torques_group, and Sellimonas) on d 14, phylum Firmicutes, genus Hydrogenoanaerobacterium, and Ruminococcaceae_V9D2013_group on d 28, genus unclassified_f_Ruminococcaceae, Anaerotruncus, Caproiciproducens, and Subdoligranulum on d 42 was observed in WXY group. Lachnospiraceae has been demonstrated to 


\section{Cladogram}

wheat

corn
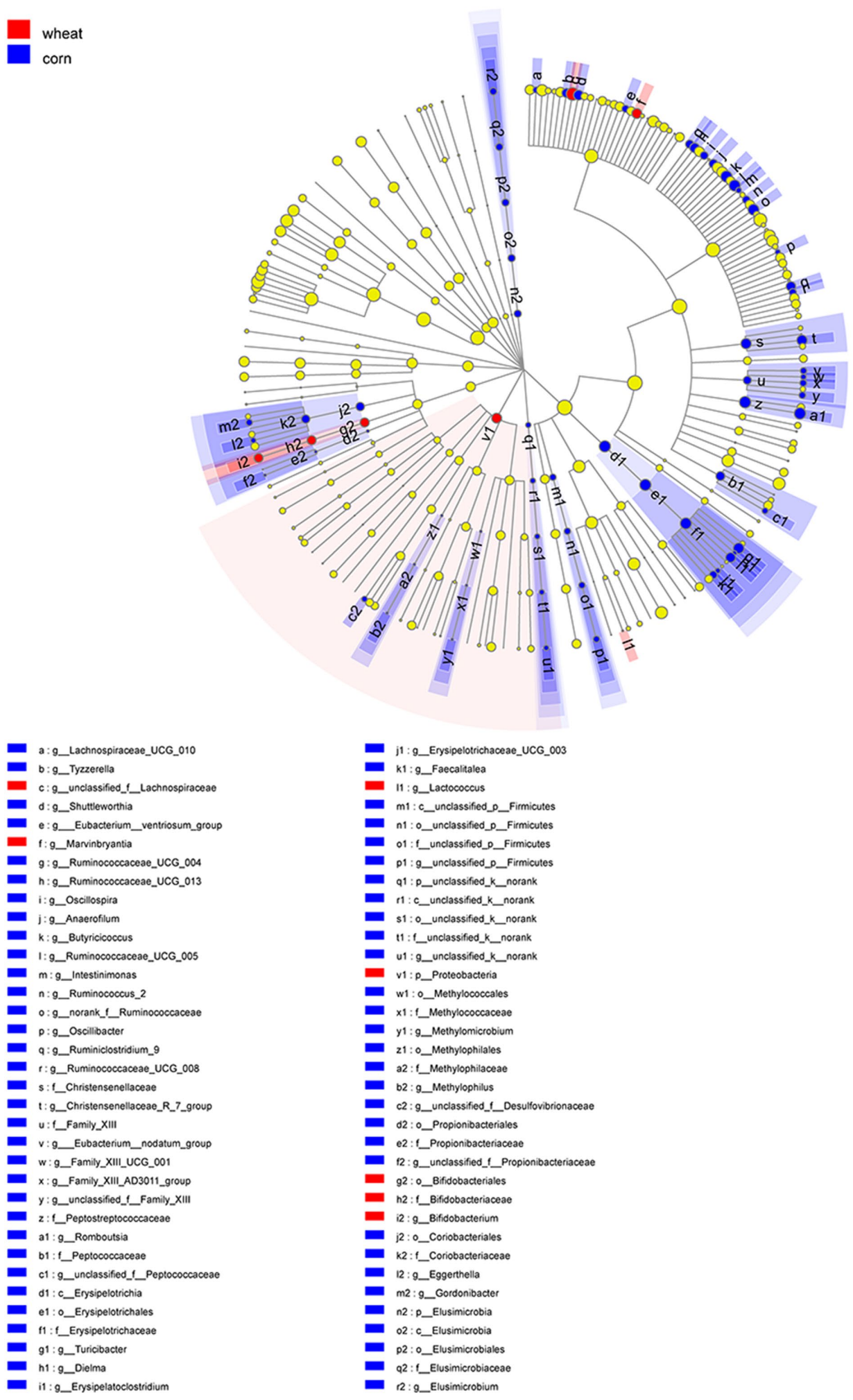

FIGURE 8 | The LEfSe analysis for cecal bacteria communities from phylum to genus level in broilers fed wheat- or corn-based diets. The circles from inner to outer represent distinct bacteria from phylum to genus level, respectively. The yellow dots inserted in the circle demonstrated no significant differences in bacteria communities between two dietary treatments. 
produce butyrate; in addition, genus norank $f_{-}$Lachnospiraceae, Shuttleworthia, and Ruminococcus_torques_group have been observed to be related to better performance (Torok et al., 2011; Lee et al., 2017; McCafferty et al., 2019a; Li et al., 2020). Genera (Hydrogenoanaerobacterium and Ruminococcaceae_V9D2013_group) from the phylum Firmicutes are also suggested to effectively produce butyrate (Fang et al., 2020; Ilaria et al., 2020; Sun et al., 2020). Genus unclassified_f_Ruminococcaceae, Anaerotruncus, Caproiciproducens, and Subdoligranulum (family Ruminococcaceae) are also known as short-chain fatty acid producers. Supplemental xylanase could modulate GIT microbiota by increasing XOS concentrations in the cecum, and XOS has been reported to increase the abundance of butyrate-producing bacteria (De Maesschalck et al., 2015; McCafferty et al., 2019a). Moreover, the present result showed that xylanase supplementation to wheat-based diets increased the abundance of Bifidobacterium (phylum Actinobacteria) on d 14, and the abundance of Lactobacillus on d 42. XOS has been demonstrated to result in better performance of broilers by the stimulation of Bifidobacterium and Lactobacillus (Oyeagu et al., 2019; González-Ortiz et al., 2020). Although some species of Actinobacteria can lead to diseases, some strains like Streptomyces spp. exhibited antibacterial activities (Jing et al., 2018). Bifidobacterium as lactate-producing bacteria benefits to the health of GIT (Jing et al., 2018; González-Ortiz et al., 2020). Members of Lactobacillus could stimulate butyrate-producing bacteria and inhibit the colonization of pathogenic bacteria (Munyaka et al., 2016; Proszkowiec-Weglarz et al., 2020). When comparing cecal microbiota of broilers fed corn-based diets, compared with CCON, higher abundance of order Clostridiales on d 28, genus Anaerofustis, Lachnospiraceae_UCG_010, and norank_f__Ruminococcaceae on d 42 was observed in broilers from CXY group. Clostridium-related bacteria within GIT have been demonstrated to be able to produce short-chain fatty acids and act as a barrier against invasion by other potentially pathogenic microbiota (Munyaka et al., 2016). The increase of these butyrate-producing bacteria might also be related to increased XOS concentrations in the cecum.

Furthermore, compared with CCON group, lower abundance of family Christensenellaceae, Desulfovibrionaceae, and genus Faecalitalea, Escherichia_Shigella on d 14, and higher abundance of family Erysipelotrichaceae on d 42 were observed in CXY group. Xylanase supplementation to wheatbased diets could also increase the abundance of genus Gordonibacter on d 14 and d 42 . The family Christensenellaceae was negatively associated with overall GIT health (Richards et al., 2019). Faecalitalea which affiliates to Erysipelotrichaceae family was related to inflammatory disorders (Dai et al., 2020). In addition, the previous work has reported that the abundance of Desulfovibrio (family Desulfovibrionaceae) was increased with ulcerative colitis, indicating that Desulfovibrio was enriched in an inflammatory environment (Yan et al., 2019). Enterobacteriaceae family includes potential pathogens, such as Escherichia_Shigella, the abundances of Escherichia Shigella were found to be negatively correlated with growth performance in broilers (Díaz Carrasco et al., 2018). Moreover, Erysipelotrichaceae showed a positive correlation with growth performance (Stanley et al., 2016). Gordonibacter was associated with a decrease risk or severity of different diseases, such as cancer and inflammatory bowel disease (Pérez-Burillo et al., 2018). Overall, the results indicated that xylanase supplementation could stimulate the colonization of beneficial bacteria or inhibit the colonization of harmful bacteria in broilers receiving wheat- or corn-based diets.

Additionally, we compared the cecal microbiota of broilers supplemented corn- or wheat-based diets. The abundance of Lactobacillus and Bifidobacterium increased in the cecal microbiota of broilers receiving wheat-based diets, and the increase may be associated with high contents of $\mathrm{AX}$ in wheat. The cecal fermentative ability of broilers depends on type and content of substrates flowing into cecum. Members of Lactobacillus contained a range of genes coding ford AX-degrading enzymes; therefore, they can be beneficial to the degradation of XOS and AX (Zhang et al., 2018b). It also has been reported that AX and XOS selectively increased the abundance of Bifidobacterium spp., a so-called bifidogenic effect exerted by these prebiotics. Also, Bifidobacterium spp. have been reported to contain large number of genes coding for AX-degrading enzymes (Rivière et al., 2014). Overall, AX and XOS are preferred substrates of Lactobacillus and Bifidobacterium, and the growth of Lactobacillus and Bifidobacterium was stimulated by wheat relative to corn.

In corn-based diets fed broilers, the abundance of butyrateproducing bacterium increased, such as Lachnospiraceae UCG_010, Shuttleworthia, Anaerofilum, Ruminococcaceae UCG_008, Ruminococcaceae_UCG_005, Ruminococcus_2, norank_f_Ruminococcaceae, Oscillibacter, and Ruminiclostridium_9 comparing to the broilers fed wheat-based diets. This difference may be attributed to the resistant starch content in corn and wheat. Corn contains the content of resistant starch 1.9-fold higher than that in wheat. Resistant starch is fermented by microbiota in large intestine and relates to increased abundance of butyrate-producing bacteria (Bednar et al., 2001; Yang et al., 2017b). Moreover, resistant starch-rich diet was associated with increased circulating propionate concentration in mice (Bai et al., 2021). In the current study, higher abundance of propionic acid-producing bacteria Propionibacteriaceae was likely to relate to the higher content of resistant starch in corn.

In wheat-based diets fed broilers, the abundance of Proteobacteria was higher than those fed corn-based diets, which may relate to proteinaceous substrates since wheatbased diets contain about $1 \%$ more protein than corn-based diets in this study. Proteobacteria are mainly responsible for utilization of amino acids and possess extensive gene coverage of amino acid responses (Dai et al., 2015; Diether and Willing, 2019). Moreover, the reason that higher abundance of Peptostreptococcaceae in the broilers fed cornbased diets was likely due to the different amino acid compositions of corn protein from wheat protein. Zein as the major fraction of corn protein is rich in leucine 
(Cowieson, 2005). Peptostreptococcus spp. was associated with leucine metabolism in hindgut ( $\mathrm{Ma}$ and Ma, 2018).

\section{CONCLUSION}

Overall, AT-xynA supplementation to wheat- or corn-based diets modulated the relative abundance of specific bacteria without changing the overall microbial structure. We showed that bacterial community clustering was mainly due to cereal grain source rather than xylanase supplementation. The addition of xylanase to wheat-based diets increased the abundance of Lactobacillus, Bifidobacterium, and some butyrate-producing bacteria, the increase might be related to the release of XOS as a result of AX degradation in GIT. Moreover, the undigested polysaccharides flowing into the cecum resulted in higher abundance of (non-starch polysaccharides) NSP-degrading bacteria in broilers fed wheat-based diets without xylanase supplementation, such as Ruminococcaceae and Bacteroidetes. Xylanase supplementation to corn-based diets slightly affected the abundance of butyrate-producing bacteria and NSP-degrading bacterium, the difference might be related to AX concentration and composition in corn and wheat. However, xylanase still increased the abundance of beneficial bacteria and decreased the abundance of harmful bacteria in the cecum of broilers fed corn-based diets. Moreover, beneficial effects of xylanase on cecal microbiome improved growth performance.

\section{REFERENCES}

Bai, Y., Li, Y., Marion, T., Tong, Y., Zaiss, M. M., Tang, Z., et al. (2021). Resistant starch intake alleviates collagen-induced arthritis in mice by modulating gut microbiota and promoting concomitant propionate production. J. Autoimmun. 116:102564. doi: 10.1016/j.jaut.2020.102564

Bautil, A., Verspreet, J., Buyse, J., Goos, P., Bedford, M. R., and Courtin, C. M. (2019). Age-related arabinoxylan hydrolysis and fermentation in the gastrointestinal tract of broilers fed wheat-based diets. Poult. Sci. 98, 4606-4621. doi: $10.3382 / \mathrm{ps} /$ pez159

Bednar, G. E., Patil, A. R., Murray, S. M., Grieshop, C. M., Merchen, N. R., and Fahey, G. C. Jr. (2001). Starch and fiber fractions in selected food and feed ingredients affect their small intestinal digestibility and fermentability and their large bowel fermentability in vitro in a canine model. J. Nutr. 131, 276-286. doi: 10.1093/jn/131.2.276

Biddle, A., Stewart, L., Blanchard, J., and Leschine, S. (2013). Untangling the genetic basis of fibrolytic specialization by Lachnospiraceae and Ruminococcaceae in diverse gut communities. Diversity 5, 627-640. doi: 10.3390/d5030627

Cao, Y. H., Qiao, J. Y., Li, Y. H., and Lu, W. Q. (2007). De novo synthesis, constitutive expression of aspergillus sulphureus $\beta$-xylanase gene in Pichia pastoris and partial enzymic characterization. Appl. Microbiol. Biotechnol. 76, 579-585. doi: 10.1007/s00253-007-0978-9

Chakraborti, C. K. (2015). New-found link between microbiota and obesity. World J. Gastrointest. Pathophysiol. 6:110. doi: 10.4291/wjgp.v6.i4.110

Choct, M., Hughes, R. J., and Bedford, M. R. (1999). Effects of a xylanase on individual bird variation, starch digestion throughout the intestine, and ileal and caecal volatile fatty acid production in chickens fed wheat. Brit. Poult. Sci. 40, 419-422. doi: 10.1080/00071669987548

Cowieson, A. J. (2005). Factors that affect the nutritional value of maize for broilers. Anim. Feed Sci. Technol. 119, 293-305. doi: 10.1016/j. anifeedsci.2004.12.017

\section{DATA AVAILABILITY STATEMENT}

The datasets presented in this study can be found in online repositories. The names of the repository/repositories and accession number(s) can be found at: https://www.ncbi.nlm. nih.gov/, PRJNA753623.

\section{ETHICS STATEMENT}

The animal study was reviewed and approved by the Laboratory Animal Welfare and Animal Experimental Ethical Inspection Committee of China Agricultural University.

\section{AUTHOR CONTRIBUTIONS}

YC and SL supervised and provided continuous guidance for the experiments. $\mathrm{YC}, \mathrm{BD}$, and $\mathrm{CW}$ conceived and designed the experiments. JW, $\mathrm{HC}, \mathrm{CB}$, and YL performed the animal experiments. JW, ZS, and $\mathrm{BD}$ analyzed the data and wrote the manuscript. All authors have discussed the results and reviewed the manuscript.

\section{FUNDING}

Our study was supported by the funds of National Natural Science Foundation of People's Republic of China (no. 31760673) and the Project of introducing talents to Tibet (RXYJ-2019-06).

Cowieson, A. J. (2010). Strategic selection of exogenous enzymes for corn/ soy-based poultry diets. J. Poult. Sci. 47, 1-7. doi: 10.2141/jpsa.009045

Dai, D., Qiu, K., Zhang, H., Wu, S., Han, Y., Wu, Y., et al. (2021). Organic acids as alternatives for antibiotic growth promoters alter the intestinal structure and microbiota and improve the growth performance in broilers. Front. Microbiol. 11:618144. doi: 10.3389/fmicb.2020.618144

Dai, Z., Wu, Z., Hang, S., Zhu, W., and Wu, G. (2015). Amino acid metabolism in intestinal bacteria and its potential implications for mammalian reproduction. Mol. Hum. Reprod. 21, 389-409. doi: 10.1093/molehr/gav003

Dai, D., Wu, S., Zhang, H., Qi, G., and Wang, J. (2020). Dynamic alterations in early intestinal development, microbiota and metabolome induced by in ovo feeding of L-arginine in a layer chick model. J. Anim. Sci. Biotechnol. 11:19. doi: 10.1186/s40104-020-0427-5

De Maesschalck, C., Eeckhaut, V., Maertens, L., De Lange, L., Marchal, L., Nezer, C., et al. (2015). Effects of xylo-oligosaccharides on broiler chicken performance and microbiota. Appl. Environ. Microb. 81, 5880-5888. doi: 10.1128/AEM.01616-15

Dhillon, J., Li, Z., and Ortiz, R. M. (2019). Almond snacking for 8 wk increases alpha-diversity of the gastrointestinal microbiome and decreases Bacteroides fragilis abundance compared with an isocaloric snack in college freshmen. Curr. Dev. Nutr. 3:z79. doi: 10.1093/cdn/nzz079

Díaz Carrasco, J. M., Redondo, E. A., Pin Viso, N. D., Redondo, L. M., Farber, M. D., and Fernández Miyakawa, M. E. (2018). Tannins and bacitracin differentially modulate gut microbiota of broiler chickens. Biomed. Res. Int. 2018, 1-11. doi: 10.1155/2018/1879168

Diether, N. E., and Willing, B. P. (2019). Microbial fermentation of dietary protein: an important factor in diet-microbe-host interaction. Microorganisms. 7:19. doi: 10.3390/microorganisms7010019

Fang, S., Chen, X., Pan, J., Chen, Q., Zhou, L., Wang, C., et al. (2020). Dynamic distribution of gut microbiota in meat rabbits at different growth stages and relationship with average daily gain (ADG). BMC Microbiol. 20:116. doi: $10.1186 / \mathrm{s} 12866-020-01797-5$ 
González-Ortiz, G., Olukosi, O. A., Jurgens, G., Apajalahti, J., and Bedford, M. R. (2020). Short-chain fatty acids and ceca microbiota profiles in broilers and turkeys in response to diets supplemented with phytase at varying concentrations, with or without xylanase. Poult. Sci. 99, 2068-2077. doi: 10.1016/j.psj.2019.11.051

Gonzalez-Ortiz, G., Sola-Oriol, D., Martinez-Mora, M., Perez, J. F., and Bedford, M. R. (2017). Response of broiler chickens fed wheat-based diets to xylanase supplementation. Poult. Sci. 96, 2776-2785. doi: 10.3382/ps/ pex092

Ilaria, B., Ilario, F., Sihem, D., Rocchina, E., Francesco, G., Laura, G., et al. (2020). Black soldier fly and gut health in broiler chickens: insights into the relationship between cecal microbiota and intestinal mucin composition. J. Anim. Sci. Biotechnol. 11:11. doi: 10.1186/s40104-019-0413-y

Jing, Y., Li, A., Liu, Z., Yang, P., Wei, J., Chen, X., et al. (2018). Absorption of Codonopsis pilosula saponins by coexisting polysaccharides alleviates gut microbial dysbiosis with dextran sulfate sodium-induced colitis in model mice. Biomed. Res. Int. 2018, 1-18. doi: 10.1155/2018/1781036

Khadem, A., Lourenco, M., Delezie, E., Maertens, L., Goderis, A., Mombaerts, R., et al. (2016). Does release of encapsulated nutrients have an important role in the efficacy of xylanase in broilers? Poult. Sci. 95, 1066-1076. doi: 10.3382/ ps/pew002

Kiarie, E., Romero, L. F., and Ravindran, V. (2014). Growth performance, nutrient utilization, and digesta characteristics in broiler chickens fed corn or wheat diets without or with supplemental xylanase. Poult. Sci. 93, 1186-1196. doi: 10.3382/ps.2013-03715

Lee, K., Kil, D. Y., and Sul, W. J. (2017). Cecal microbiome divergence of broiler chickens by sex and body weight. J. Microbiol. 55, 939-945. doi: 10.1007/s12275-017-7202-0

Li, H., Zhang, H., Zhao, F., Wang, S., Wang, Z., and Wei, Z. (2020). Modulation of gut microbiota, short-chain fatty acid production, and inflammatory cytokine expression in the cecum of porcine Deltacoronavirus-infected chicks. Front. Microbiol. 11:897. doi: 10.3389/fmicb.2020.00897

Liu, J., Stewart, S. N., Robinson, K., Yang, Q., Lyu, W., Whitmore, M. A., et al. (2021). Linkage between the intestinal microbiota and residual feed intake in broiler chickens. J. Anim. Sci. Biotechnol. 12:22. doi: 10.1186/ s40104-020-00542-2

Liu, W. C., and Kim, I. H. (2017). Effects of dietary xylanase supplementation on performance and functional digestive parameters in broilers fed wheatbased diets. Poult. Sci. 96, 566-573. doi: 10.3382/ps/pew258

Ma, N., and Ma, X. (2018). Dietary amino acids and the gut-microbiomeimmune axis: physiological metabolism and therapeutic prospects. Compr. Rev. Food Sci. Food Saf. 18, 221-242. doi: 10.1111/1541-4337.12401

Ma, Z. Y., Zhang, X. M., Wang, M., Wang, R., Jiang, Z. Y., Tan, Z. L., et al. (2019). Molecular hydrogen produced by elemental magnesium inhibits rumen fermentation and enhances methanogenesis in dairy cows. J. Dairy Sci. 102, 5566-5576. doi: 10.3168/jds.2018-15647

Masey-O'Neill, H. V., Singh, M., and Cowieson, A. J. (2014). Effects of exogenous xylanase on performance, nutrient digestibility, volatile fatty acid production and digestive tract thermal profiles of broilers fed on wheat- or maize-based diet. Brit. Poult. Sci. 55, 351-359. doi: 10.1080/00071668.2014.898836

McCafferty, K. W., Bedford, M. R., Kerr, B. J., and Dozier, W. A. (2019a). Effects of age and supplemental xylanase in corn- and wheat-based diets on cecal volatile fatty acid concentrations of broilers. Poult. Sci. 98, 4787-4800. doi: $10.3382 / \mathrm{ps} / \mathrm{pez} 194$

McCafferty, K. W., Bedford, M. R., Kerr, B. J., and Dozier, W. A. (2019b). Effects of cereal grain source and supplemental xylanase concentrations on broiler growth performance and cecal volatile fatty acid concentrations from 1 to $40 \mathrm{~d}$ of age. Poult. Sci. 98, 2866-2879. doi: 10.3382/ps/pez032

Munyaka, P. M., Nandha, N. K., Kiarie, E., Nyachoti, C. M., and Khafipour, E. (2016). Impact of combined beta-glucanase and xylanase enzymes on growth performance, nutrients utilization and gut microbiota in broiler chickens fed corn or wheat-based diets. Poult. Sci. 95, 528-540. doi: 10.3382/ps/ pev333

National Research Council (1994). Nutrient Requirements of Poultry. 9th Edn. Washington, DC: The National Academies Press.

Ocejo, M., Oportp, B., and Hurtado, A. (2019). 16S rRNA amplicon sequencing characterization of caecal microbiome composition of broilers and free-range slow-growing chickens throughout their productive lifespan. Sci. Rep. 9:2506. doi: 10.1038/s41598-019-39323-x
Onrust, L., Ducatelle, R., Van Driessche, K., De Maesschalck, C., Vermeulen, K., Haesebrouck, F., et al. (2015). Steering endogenous butyrate production in the intestinal tract of broilers as a tool to improve gut health. Front. Vet. Sci. 2:75. doi: $10.3389 /$ fvets.2015.00075

Oyeagu, C. E., Mlambo, V., Muchenje, V., and Marume, U. (2019). Effect of dietary supplementation of aspergillus xylanase on broiler chickens performance. Iranian J. Appl. Anim. Sci. 9, 693-708.

Pérez-Burillo, S., Pastoriza, S., Jiménez-Hernández, N., Auria, D. G., Francino, M. P., and Rufián-Henares, J. A. (2018). Effect of food thermal processing on the composition of the gut microbiota. J. Agr. Food Chem. 66, 11500-11509. doi: $10.1021 /$ acs.jafc.8b04077

Petra, V., Karel, S., Maja, L., Marcela, F., Lenka, G., Darina, C., et al. (2014). Succession and replacement of bacterial populations in the caecum of egg laying hens over their whole life. PLoS One 9:e115142. doi: 10.1371/journal. pone. 0115142

Petry, A. L., Patience, J. F., Nichole, F. H., Koester, L. R., Bedford, M. R., and Schmitz-Esser, S. (2021). Xylanase supplementation modulates the microbiota of the large intestine of pigs fed corn-based fiber by means of a stimbiotic mechanism of action. Front. Microbiol. 12:619970. doi: 10.3389/fmicb.2021.619970

Polansky, O., Sekelova, Z., Faldynova, M., Sebkova, A., Sisak, F., and Rychlik, I. (2016). Important metabolic pathways and biological processes expressed by chicken cecal microbiota. Appl. Environ. Microb. 82, 1569-1576. doi: 10.1128/AEM.03473-15

Proszkowiec-Weglarz, M., Miska, K. B., Schreier, L. L., Grim, C. J., Jarvis, K. G., Shao, J., et al. (2020). Effect of butyric acid glycerol esters on ileal and cecal mucosal and luminal microbiota in chickens challenged with Eimeria maxima. Poult. Sci. 99, 5143-5148. doi: 10.1016/j.psj.2020.06.022

Richards, P., Fothergill, J., Bernardeau, M., and Wigley, P. (2019). Development of the caecal microbiota in three broiler breeds. Front. Vet. Sci. 6:201. doi: 10.3389/fvets.2019.00201

Ríos-Covián, D., Ruas-Madiedo, P., Margolles, A., Gueimonde, M., de Los Reyes-Gavilán, C. G., and Salazar, N. (2016). Intestinal short chain fatty acids and their link with diet and human health. Front. Microbiol. 7:185. doi: $10.3389 /$ fmicb.2016.00185

Rivière, A., Moens, F., Selak, M., Maes, D., Weckx, S., and De Vuyst, L. (2014). The ability of Bifidobacteria to degrade arabinoxylan oligosaccharide constituents and derived oligosaccharides is strain dependent. Appl. Environ. Microb. 80, 204-217. doi: 10.1128/AEM.02853-13

Shang, Y., Kumar, S., Oakley, B., and Kim, W. K. (2018). Chicken gut microbiota: importance and detection technology. Front. Vet. Sci. 5:254. doi: 10.3389/ fvets.2018.00254

Stanley, D., Hughes, R. J., Geier, M. S., and Moore, R. J. (2016). Bacteria within the gastrointestinal tract microbiota correlated with improved growth and feed conversion: challenges presented for the identification of performance enhancing probiotic bacteria. Front. Microbiol. 7:187. doi: 10.3389/ fmicb.2016.00187

Sun, X., Shen, J., Liu, C., Li, S., Peng, Y., Chen, C., et al. (2020). L-arginine and N-carbamoylglutamic acid supplementation enhance young rabbit growth and immunity by regulating intestinal microbial community. Asian Austral. J. Anim. 33, 166-176. doi: 10.5713/ajas. 18.0984

Torok, V. A., Hughes, R. J., Mikkelsen, L. L., Perez-Maldonado, R., Balding, K., MacAlpine, R., et al. (2011). Identification and characterization of potential performance-related gut microbiotas in broiler chickens across various feeding trials. Appl. Environ. Microb. 77, 5868-5878. doi: 10.1128/AEM.00165-11

Wang, J., Liu, Y., Yang, Y., Bao, C., and Cao, Y. (2020). High-level expression of an acidic thermostable xylanase in Pichia pastoris and its application in weaned piglets. J. Anim. Sci. 98:skz364. doi: 10.1093/jas/skz364

Wang, J., Liu, S. J., Ma, J. Y., and Piao, X. S. (2021). Changes in growth performance and Ileal microbiota composition by Xylanase supplementation in broilers fed wheat-based diets. Front. Microbiol. 12:706396. doi: 10.3389/ fmicb.2021.706396

Yadav, S., and Jha, R. (2019). Strategies to modulate the intestinal microbiota and their effects on nutrient utilization, performance, and health of poultry. J. Anim. Sci Biotechnol. 10:2. doi: 10.1186/s40104-018-0310-9

Yan, J., Zhou, B., Xi, Y., Huan, H., Li, M., Yu, J., et al. (2019). Fermented feed regulates growth performance and the cecal microbiota community in geese. Poult. Sci. 98, 4673-4684. doi: 10.3382/ps/pez169

Yang, J., Martinez, I., Walter, J., Keshavarzian, A., and Rose, D. J. (2013). In vitro characterization of the impact of selected dietary fibers on fecal 
microbiota composition and short chain fatty acid production. Anaerobe 23, 74-81. doi: 10.1016/j.anaerobe.2013.06.012

Yang, W., Yang, Y., Zhang, L., Xu, H., Guo, X., Yang, X., et al. (2017a). Improved thermostability of an acidic xylanase from aspergillus sulphureus by combined disulphide bridge introduction and proline residue substitution. Sci. Rep. 7:1587. doi: 10.1038/s41598-017-01758-5

Yang, X., Darko, K. O., Huang, Y., He, C., Yang, H., He, S., et al. (2017b). Resistant starch regulates gut microbiota: structure, biochemistry and cell signalling. Cell. Physiol. Biochem. 42, 306-318. doi: 10.1159/000477386

Zhang, Z., Tun, H. M., Li, R., Gonzalez, B. J. M., Keenes, H. C., Nyachoti, C. M., et al. (2018a). Impact of xylanases on gut microbiota of growing pigs fed corn- or wheat-based diets. Anim. Nutr. 4, 339-350. doi: 10.1016/j.aninu.2018.06.007

Zhang, Y. J., Liu, Q., Zhang, W. M., Zhang, Z. J., Wang, W. L., and Zhuang, S. (2018b). Gastrointestinal microbial diversity and short-chain fatty acid production in pigs fed different fibrous diets with or without cell walldegrading enzyme supplementation. Livest. Sci. 207, 105-116. doi: 10.1016/j. livsci.2017.11.017
Conflict of Interest: The authors declare that the research was conducted in the absence of any commercial or financial relationships that could be construed as a potential conflict of interest.

Publisher's Note: All claims expressed in this article are solely those of the authors and do not necessarily represent those of their affiliated organizations, or those of the publisher, the editors and the reviewers. Any product that may be evaluated in this article, or claim that may be made by its manufacturer, is not guaranteed or endorsed by the publisher.

Copyright (c) 2021 Wang, Cao, Bao, Liu, Dong, Wang, Shang, Cao and Liu. This is an open-access article distributed under the terms of the Creative Commons Attribution License (CC BY). The use, distribution or reproduction in other forums is permitted, provided the original author(s) and the copyright owner(s) are credited and that the original publication in this journal is cited, in accordance with accepted academic practice. No use, distribution or reproduction is permitted which does not comply with these terms. 